\title{
Existence and concentration of positive solutions for a class of discontinuous quasilinear Schrödinger problems in $\mathbb{R}^{N}$
}

\section{Ziqing Yuan ${ }^{1,2^{*}}$}

\section{Correspondence:}

312917335@163.com

'Department of Mathematics,

Shaoyang University, Shaoyang, P.R. China

${ }^{2}$ Center for Applied Mathematics, Guangzhou University, Guangzhou, P.R. China

\section{Springer}

\begin{abstract}
In this paper, a class of quasilinear Schrödinger equations with discontinuous nonlinearity is considered. After changing variables, by using nonsmooth critical point theory, we obtain the existence and concentration of positive solutions for this problem under suitable conditions. Our results cover and extend some results for these differentiable quasilinear Schrödinger problems.
\end{abstract}

MSC: $35 J 85 ; 47 J 30 ; 49 J 52$

Keywords: Quasilinear Schrödinger equation; Variational method; Discontinuous nonlinearity; Positive solution

\section{Introduction}

Recently many papers [1-5] have focused on studying the existence of solutions for the following quasilinear Schrödinger equations:

$$
i \epsilon \frac{\partial \psi}{\partial t}=-\epsilon^{2} \Delta \psi+W(x) \psi-\epsilon^{2} k \Delta\left(h\left(|\psi|^{2}\right)\right) h^{\prime}\left(|\psi|^{2}\right) \psi-g\left(|\psi|^{2}\right) \psi, \quad x \in \mathbb{R}^{N},
$$

where $\epsilon>0, W$ is a given potential, $k \in \mathbb{R}, g$ and $h$ are real functions. Equation (1.1) with various types of $h$ appears in several areas of physics. For example, in the case $h(s)=(1+s)^{\frac{1}{2}}$, problem (1.1) models the self-channeling of a high-power ultra-short laser in matter, the propagation of a high-irradiance laser in a plasma creates an optical index depending nonlinearly on the light intensity and this leads to interesting new nonlinear wave equations [6, 7]. For more applications, we can refer to [8-10] and the references therein. Here, we are interested in studying the case $h(s)=s$, which is used to model a superfluid film in plasma physics [4], especially the existence of standing wave solutions, that is, solutions of type $\psi=\exp (-i E t / \epsilon) u(x)$ with $E \in \mathbb{R}$ and function $u>0$ [11-13]. After a direct computation, problem (1.1) is equivalent to

$$
-\Delta u+V(\epsilon x) u-k \Delta\left(u^{2}\right) u=g(u), \quad x \in \mathbb{R}^{N} .
$$

(c) The Author(s) 2020. This article is licensed under a Creative Commons Attribution 4.0 International License, which permits use, sharing, adaptation, distribution and reproduction in any medium or format, as long as you give appropriate credit to the original author(s) and the source, provide a link to the Creative Commons licence, and indicate if changes were made. The images or other third party material in this article are included in the article's Creative Commons licence, unless indicated otherwise in a credit line to the material. If material is not included in the article's Creative Commons licence and your intended use is not permitted by statutory regulation or exceeds the permitted use, you will need to obtain permission directly from the copyright holder. To view a copy of this licence, visit http://creativecommons.org/licenses/by/4.0/ 
It is well known that there exist lots of results on discussing Eq. (1.2) with $k=0$, i.e., the following semilinear case:

$$
-\Delta u+V(\epsilon x) u=g(u), \quad x \in \mathbb{R}^{N} .
$$

In [14] Rabinowitz used the mountain pass theorem to prove the existence of positive solutions of (1.3) for $\epsilon>0$ and $V$ satisfying

(V0) $V_{\infty}=\liminf _{|x| \rightarrow \infty} V(x)>\inf _{x \in \mathbb{R}^{N}} V(x)=m>0$.

Later, Alves and Figueiredo [15] extended (1.3) to the $p$-Laplace case with $2 \leq p<N$ and proved that these solutions concentrate at global minimum points of $V(\epsilon x)$ as $\epsilon \rightarrow 0$. More results can be found in [16-20] and so on.

Compared to the semilinear case, the quasilinear case $(k \neq 0)$ becomes much more complicated as there is no suitable space for the energy functional corresponding to problem (1.2) for $N \geq 2$. In order to overcome this difficulty, in [21], by changing of variables, the authors reduced the quasilinear equation (1.2) into the semilinear case. Based on this fact, problem (1.2) has been widely studied by assuming different hypotheses on $V$ and $f$. Moameni [22] obtained the existence of a positive solution by assuming that $f$ is a nonnegative function for $N \geq 2$, and the potential function $V$ is radially symmetric. Miyagaki and Moreira [11] derived the existence and multiplicity of solutions for problem (1.2) when the nonlinearity is indefinite in sign. Liu et al. $[12,13]$ developed a perturbation method, the main idea of which is adding a regularizing term to recover the smoothness of the energy functional, so that the standard minimax theory can be used. Utilizing this method and a constrained minimization argument, they proved that problem (1.2) has a positive solution. Later, $\mathrm{Wu}$ [23] showed the existence of high energy solutions by employing the perturbation method for a general quasilinear problem. Recently, Carrião et al. [1] investigated the existence of a least energy solution for a class of nonhomogeneous asymptotically linear Schrödinger equations in $\mathbb{R}^{N}$ via the Pohozaev manifold. It is worth to point out that different from semilinear problems, the critical exponent of problem (1.2) is $22^{*}$, not $2^{*}$, where $22^{*}=\frac{4 N}{N-2}$. This will lead to some difficulties. For example, some properties in the usual Sobolev space cannot be used directly. The behavior of $h$ at infinity plays an important role when searching for a solution to problem (1.2), mainly supercritical, critical or subcritical cases, where $h$ behaves at infinity as $|s|^{r-1} s$, with $r+1>22^{*}, r+1=22^{*}$ or $r+1<22^{*}$, respectively. The critical case of (1.2) was considered in [24-27]. The supercritical results can be found in [28-39] and the references therein.

However, there seems to be little progress on the existence of positive solutions for general quasilinear elliptic equations with discontinuous nonlinearity. Based on this fact, we will study the quasilinear Schrödinger Eq. (1.2) from a discontinuous point of view. To some degree, the discontinuous case is more suitable to objective reality, and a smooth situation is usually just an ideal case. Hence, we consider the existence and concentration of solutions for the following problem:

$$
\left\{\begin{array}{l}
-\Delta u-\Delta\left(u^{2}\right) u+V(\epsilon x) u=H(u-a) u^{p}, \quad x \in \mathbb{R}^{N}, \\
u>0
\end{array}\right.
$$

where $\epsilon, \beta>0$ are positive parameters, $p \in\left(3,22^{*}-2\right)$ if $N \geq 3$ or $p \in(3,+\infty)$ if $N=1,2$, $V \in C\left(\mathbb{R}^{N}, \mathbb{R}^{+}\right)$satisfying (V0). 
As is well known, the interest in studying nonlinear partial differential equations with discontinuous nonlinearities has increased since many free boundary problems and obstacle problems may be reduced to partial differential equations with nonsmooth potentials. Among these problems, we have the seepage surface problem, the obstacle problem, and the Elenbaas equation, see [40-42]. The area of nonsmooth analysis is closely related with the development of critical point theory for nondifferentiable functionals, in particular, for locally Lipschitz continuous functionals based on Clarke's generalized gradient [43]. In 1981, Chang [40] extended the variational method to a class of nondifferentiable functionals, and directly applied the variational method to prove some existence of theorems for PDE with discontinuous nonlinearities. It provides an appropriate mathematical framework to extend the classic critical point theory for $C^{1}$-functionals in a natural way, and to meet specific needs in applications, such as nonsmooth mechanics and engineering. For a comprehensive understanding, we refer to Refs. [44-53].

This paper mainly discusses the existence of positive solutions to problem (1.4). Contrast to the previous results, our methods are totally different from those used in previous papers, since we are dealing with a discontinuous and non-convex problem. The main differences are the following:

(1) Unlike [1], the lack of differentiability of nonlinearities causes some technical difficulties. This means that variational methods for $C^{1}$ functionals are not suitable in our case, since in our case, the energy functional is only locally Lipschitz continuous. Therefore, we have to use another variational approach based on the nonsmooth critical point theory due to Clarke [43] and Chang [54]. In contrast to $C^{1}$ variational methods, this method is not adequately developed, and we need to improve it.

(2) In [1], if the energy functional associated to problem (1.2) is differentiable, it can be discussed on the Nehari manifold and the mountain pass level is equal to the minimum of the energy functional on Nehari manifolds, which is a key point in lots of papers. However, all these properties are not true for nondifferentiable problems. Hence, the arguments used in the above references cannot be directly repeated and we need to develop some new techniques to get over these difficulties.

(3) Due to the appearance of the non-convex term $\Delta\left(u^{2}\right) u$, some arguments used in standard semilinear problems cannot be used, therefore lots of estimates in this paper need to be reestablished.

(4) Since $H^{1}\left(\mathbb{R}^{N}\right) \hookrightarrow L^{p}\left(\mathbb{R}^{N}\right)\left(p \in\left[2,2^{*}\right]\right)$ is not compact, and the compact embedding is very crucial to deduce (PS) sequences in variational methods, we have to use other means to overcome this difficulty.

The main result is the following.

Theorem 1.1 If hypothesis (V0) holds, then there exist $\epsilon^{*}, a^{*}>0$ such that problem (1.4) has a positive solution $u_{\epsilon, a}$ for $\epsilon \in\left(0, \epsilon^{*}\right)$ and $a \in\left(0, a^{*}\right)$. Furthermore, if $y_{\epsilon, a} \in \mathbb{R}^{N}$ denotes a maximum point of $u_{\epsilon, a}$, we have

$$
\lim _{(\epsilon, a) \rightarrow(0,0)} V\left(\epsilon y_{\epsilon, a}\right)=m
$$

Our paper is organized as follows. In Sect. 2, we give some basic results involving locally Lipschitz continuous functionals. In Sect. 3, we deal with the existence of solutions for an auxiliary problem. Then we prove Theorem 1.1 in Sect. 4 . 


\section{Preliminary results}

In the sequel, we will use the following basic notations.

- $\rightarrow$ means weak convergence while $\rightarrow$ means strong convergence.

- $C$ and $C_{i}(i=1,2, \ldots)$ denote estimated constants (the exact value may be different from line to line). $o_{n}(1)$ denotes a sequence whose limit is 0 as $n \rightarrow \infty$.

- $(X,\|\cdot\|)$ denotes a (real) Banach space and $\left(X^{*},\|\cdot\|_{*}\right)$ denotes its topological dual, $|\cdot|_{r}$ denotes the norm of $L^{r}\left(\mathbb{R}^{N}\right)$.

Definition 2.1 ([43]) A function $I: X \rightarrow \mathbb{R}$ is locally Lipschitz if for every $u \in X$ there exist a neighborhood $U$ of $u$ and $L>0$ such that for every $v, \eta \in U$

$$
|I(v)-I(\eta)| \leq L\|v-\eta\|
$$

Definition 2.2 ([43]) Let $I: X \rightarrow \mathbb{R}$ be a locally Lipschitz function. The generalized derivative of $I$ in $u$ along the direction $v$ is defined by

$$
I^{0}(u ; v)=\limsup _{\eta \rightarrow u, \tau \rightarrow 0^{+}} \frac{I(\eta+\tau \nu)-I(\eta)}{\tau},
$$

where $u, v \in X$.

It is easy to see that the function $v \mapsto I^{0}(u ; v)$ is sublinear, continuous and so is the support function of a nonempty, convex and $w^{*}$-compact set $\partial I(u) \subset X^{*}$, defined by

$$
\partial I(u)=\left\{u^{*} \in X^{*}:\left\langle u^{*}, v\right\rangle_{X} \leq I^{0}(u ; v) \text { for all } v \in X\right\} .
$$

If $I \in C^{1}(X)$, then

$$
\partial I(u)=\left\{I^{\prime}(u)\right\}
$$

Clearly, these definitions extend those of the Gâteaux directional derivative and gradient.

Definition 2.3 ([46])

(i) $I$ satisfies the nonsmooth (PS) $c$ condition if every sequence $\left\{u_{n}\right\} \subset X$ satisfying

$$
I\left(u_{n}\right) \rightarrow c \quad \text { and } \quad m^{I}\left(u_{n}\right) \rightarrow 0 \quad \text { as } n \rightarrow \infty,
$$

has a strongly convergent subsequence, where $m^{I}\left(u_{n}\right)=\inf _{u_{n}^{*} \in \partial I\left(u_{n}\right)}\left\|u_{n}^{*}\right\|_{X^{*}}$.

(ii) $I$ satisfies the nonsmooth C-condition if every sequence $\left\{u_{n}\right\} \subset X$ satisfying

$$
I\left(u_{n}\right) \rightarrow c \quad \text { and } \quad\left(1+\left\|u_{n}\right\|\right) m^{I}\left(u_{n}\right) \rightarrow 0,
$$

has a strongly convergent subsequence, where $m^{I}\left(u_{n}\right)=\inf _{u_{n}^{*} \in \partial I\left(u_{n}\right)}\left\|u_{n}^{*}\right\|_{X^{*}}$.

Proposition 2.1 ([43])

(i) $(-h)^{0}(u ; z)=h^{0}(u ;-z)$ for all $u, z \in X$;

(ii) $h^{0}(u ; z)=\max \left\{\left\langle u^{*}, z\right\rangle_{X}: u^{*} \in \partial h(u)\right\}$ for all $u, z \in X$; 
(iii) Let $j: X \rightarrow \mathbb{R}$ be a continuously differentiable function. Then $\partial j(u)=\left\{j^{\prime}(u)\right\}, j^{0}(u ; z)$ coincides with $\left\langle j^{\prime}(u), z\right\rangle_{X}$ and $(h+j)^{0}(u ; z)=h^{0}(u ; z)+\left\langle j^{\prime}(u), z\right\rangle_{X}$ for all $u, z \in X$;

(iv) (Lebourg's mean value theorem) Let $u$ and $v$ be two points in $X$. Then there exists a point $\xi$ in the open segment between $u$ and $v$, and $u_{\xi}^{*} \in \partial h(\omega)$ such that

$$
h(u)-h(v)=\left\langle u_{\xi}^{*}, u-v\right\rangle_{X}
$$

(v) (Second chain rule) Let $Y$ be a Banach space and $j: Y \rightarrow X$ be a continuously differentiable function. Then $h \circ j$ is locally Lipschitz and

$$
\partial(h \circ j)(y) \subseteq \partial h(j(y)) \circ j^{\prime}(y) \quad \text { for all } y \in Y
$$

(vi) $m^{h}(u)=\inf _{u^{*} \in \partial h(u)}\left\|u^{*}\right\|_{X^{*}}$ is lower semicontinuous.

Proposition $2.2([40,42])$ Let $\left\{u_{n}\right\} \subset X$ and $\left\{u_{n}^{*}\right\} \subset X^{*}$ with $u_{n}^{*} \in \partial I\left(u_{n}\right)$. If $u_{n} \rightarrow u$ in $X$ and $u_{n}^{*} \rightarrow u^{*}$ in $X^{*}$, then $u^{*} \in \partial I(u)$.

Proposition $2.3([40,42])$ Let $\Psi(u)=\int_{\mathbb{R}^{N}} G(u) d x$, where $G(t)=\int_{0}^{t} g(s) d s$. Then, $\Psi \in$ $\operatorname{Lip}_{\text {loc }}\left(L^{p+1}\left(\mathbb{R}^{N}\right), \mathbb{R}\right), \partial \Psi(u) \subset L^{\frac{p+1}{p}}\left(\mathbb{R}^{N}\right)$ and if $\rho \in \partial \Psi(u)$, it satisfies

$$
\rho(x) \in[\underline{g}(u(x)), \bar{g}(u(x))] \text { a.e. in } \mathbb{R}^{N} .
$$

Lemma 2.1 ([55]) Assume $\Phi \in \operatorname{Lip}_{\text {loc }}(X, \mathbb{R})$. Let $K$ be a compact metric space, $K_{0} \subset K$, with $K_{0} \neq \varnothing$ and $f_{0} \in C\left(K_{0}, X\right)$. Set

$$
\begin{aligned}
& \Gamma \equiv\left\{f \in C(K, X)|f|_{K_{0}}=f_{0}\right\}, \\
& c \equiv \inf _{f \in \Gamma} \max _{t \in K} \Phi(f(t)) .
\end{aligned}
$$

Assume that for each $f \in \Gamma$, there is some $t_{f} \in K \backslash K_{0}$ such that

$$
\max _{t \in K} \Phi(f(t))=\Phi\left(f\left(t_{f}\right)\right)
$$

Then there exists a sequence $u_{n} \in X$ satisfying

$$
\Phi\left(u_{n}\right) \rightarrow c \text { and } \min _{u_{n}^{*} \in \partial \Phi\left(u_{n}\right)}\left\|u_{n}^{*}\right\|_{X^{*}} \rightarrow 0
$$

\section{An auxiliary problem}

In this section, we firstly discuss an auxiliary problem, which is very important in proving Theorem 1.1. Note that weak solutions of (1.4) are critical points of the following functional:

$$
I_{\epsilon, a}(u)=\frac{1}{2} \int_{\mathbb{R}^{N}}\left(1+2|u|^{2}\right)|\nabla u|^{2} d x+\frac{1}{2} \int_{\mathbb{R}^{N}} V(\epsilon x) u^{2} d x-\int_{\mathbb{R}^{N}} G(u) d x
$$


where $G(u)=\int_{0}^{t} g(s) d s, g(t)=H(t-a) t^{p}$. While, in order to find critical points of (3.1), we need to study the existence of solutions to problem (1.4) with $\epsilon=1$, i.e.,

$$
\left\{\begin{array}{l}
-\Delta u-\Delta\left(u^{2}\right) u+V(x) u=H(u-a) u^{p}, \quad x \in \mathbb{R}^{N}, \\
u>0 .
\end{array}\right.
$$

The Euler-Lagrange functional corresponding to problem (3.2) $I_{a}: E \rightarrow \mathbb{R}$ is given by

$$
I_{a}(u)=\frac{1}{2} \int_{\mathbb{R}^{N}}\left(1+2|u|^{2}\right)|\nabla u|^{2} d x+\frac{1}{2} \int_{\mathbb{R}^{N}} V(x) u^{2} d x-\int_{\mathbb{R}^{N}} G(u) d x,
$$

where $E=\left\{u \in H^{1}\left(\mathbb{R}^{N}\right): \int_{\mathbb{R}^{N}} V(x)|u|^{2} d x<\infty\right\}$ with the norm $\|u\|^{2}=\int_{\mathbb{R}^{N}}\left(|\nabla u|^{2}+\right.$ $\left.V(x) u^{2}\right) d x$. However, from (3.3) we can see that $I_{a}$ is not well defined in general in $E$. In order to overcome this difficulty, we adopt an method developed by Liu et al. [56] and Colin and Jeanjean [21]. Make the change of variables by $u=f(v)$, where $f$ is defined by

$$
f^{\prime}(t)=\frac{1}{\sqrt{1+2 f^{2}(t)}} \quad \text { on }[0,+\infty)
$$

and

$$
f(-t)=-f(t) \quad \text { on }(-\infty, 0] .
$$

From [21], one has the following lemma.

Lemma 3.1 The function $f(t)$ and its derivative satisfy the following properties:

$(f 1) f$ is uniquely defined, $C^{\infty}(\mathbb{R})$ and invertible.

(f2) $\left|f^{\prime}(t)\right| \leq 1$ for all $t \in \mathbb{R}$.

(f3) $|f(t)| \leq|t|$ for all $t \in \mathbb{R}$.

(f4) $\frac{f(t)}{t} \rightarrow 1$ as $t \rightarrow 0$.

(f5) $\frac{f(t)}{\sqrt{t}} \rightarrow 2^{\frac{1}{4}}$ as $t \rightarrow+\infty$.

(f6) $\frac{f(t)}{2} \leq t f^{\prime}(t) \leq f(t)$ for all $t>0$.

(f7) $\frac{f^{2}(t)}{2} \leq t f^{\prime}(t) f(t) \leq f^{2}(t)$ for all $t \in \mathbb{R}$.

(f8) $|f(t)| \leq 2^{\frac{1}{4}}|t|^{\frac{1}{2}}$ for all $t \geq \mathbb{R}$.

(f9) There exists a positive constant $C$ such that

$$
|f(t)| \geq \begin{cases}C|t|, & |t| \leq 1 \\ C|t|^{\frac{1}{2}}, & |t| \geq 1\end{cases}
$$

(f10) For each $\alpha>0$, there exists a positive constant $C(\alpha)$ such that

$$
|f(\alpha t)|^{2} \leq C(\alpha)|f(t)|^{2}
$$

(f11) $\left|f(t) f^{\prime}(t)\right| \leq \frac{1}{\sqrt{2}}$

(f12) For each $\lambda>1$ and all $t \in \mathbb{R}, f^{2}(\lambda t) \leq \lambda^{2} f^{2}(t)$.

(f13) For each $\lambda<1$ and all $t \in \mathbb{R}, f^{2}(\lambda t) \geq \lambda^{2} f^{2}(t)$. 
Therefore, after the change of variable, from $I_{a}(u)$ we have the following functional

$$
J_{a}(v)=\frac{1}{2} \int_{\mathbb{R}^{N}}|\nabla v|^{2} d x+\frac{1}{2} \int_{\mathbb{R}^{N}} V(x) f^{2}(v) d x-\int_{\mathbb{R}^{N}} G(f(v)) d x
$$

where $J_{a}$ is well defined on the space $E$. Arguing as in [21], if $v$ is a critical point of the functional $J_{a}$, then $u=f(v)$ is a critical point of the functional $I_{a}$, i.e., $u=f(v)$ is a solution of problem (3.2). Since we are looking for positive solutions to problem (3.2), we only need to require $f(v)>0$, i.e., $v>0$.

Lemma 3.2 The functional $J_{a}$ satisfies the mountain pass geometry.

Proof We introduce the following notations for the functional $J_{a}$ :

$$
J_{a}(v)=Q_{1}(v)-Q_{2}(v)
$$

where $Q_{1}(v)=\frac{1}{2} \int_{\mathbb{R}^{N}}|\nabla v|^{2} d x+\frac{1}{2} \int_{\mathbb{R}^{N}} V(x) f^{2}(v) d x$ and $Q_{2}(v)=\int_{\mathbb{R}^{N}} G(f(v)) d x$. Since $Q_{1}(v)$ is a smooth continuous functions, we only need to show that $Q_{2}(v)$ is locally Lipschitz. Let $v_{1}, v_{2} \in E$. Consider

$$
\begin{aligned}
\left|Q_{2}\left(v_{2}\right)-Q_{2}\left(v_{1}\right)\right| & \leq \int_{\mathbb{R}^{N}}\left|\int_{v_{1}}^{v_{2}} f^{p}(t) f^{\prime}(t) d t\right| d x \\
& \leq \int_{\mathbb{R}^{N}}\left|\int_{v_{1}}^{v_{2}} f^{p}(t) d t\right| d x \\
& \leq \int_{\mathbb{R}^{N}}\left(f^{p}\left(v_{2}\right)+f^{p}\left(v_{1}\right)\right)\left|v_{2}(x)-v_{1}(x)\right| d x \\
& \leq 2^{\frac{p+4}{4}} \int_{\mathbb{R}^{N}}|w(x)|^{\frac{p}{2}}\left|v_{2}(x)-v_{1}(x)\right| d x \\
& \leq 2^{\frac{p+4}{4}}\left|v_{2}-v_{1}\right| \frac{p}{2}+1|w|_{\frac{p}{2}+1}^{\frac{p}{2}} \\
& \leq 2^{\frac{p+4}{4}} C\|w\|^{\frac{p}{2}}\left\|v_{2}-v_{1}\right\|,
\end{aligned}
$$

where $w(x)=\max \left\{f^{p}\left(v_{1}(x)\right), f^{p}\left(v_{2}(x)\right)\right\}$. Therefore, $Q_{2}$ is locally Lipschitz on $E$.

Setting $S(r)=\{v \in E:\|v\|=r\}$, we now show that there exist $r, \beta>0$ such that

$$
J(v) \geq \beta \quad \text { for all } v \in S(r)
$$

By $(f 3)$ in Lemma 3.1, we have

$$
\int_{\mathbb{R}^{N}}\left(|\nabla v|^{2}+V(x) f^{2}(v)\right) d x \leq \int_{\mathbb{R}^{N}}\left(|\nabla v|^{2}+V(x) v^{2}\right) d x
$$

which means that $\|v\|_{0}^{2}=\int_{\mathbb{R}^{N}}\left(|\nabla v|^{2}+V(x) f^{2}(v)\right) d x$ is bounded. Using this condition, from Lemma [57, Lemma 2.4], we have

$$
\|v\|_{0}^{2} \geq C_{1}\|v\|^{2}
$$


Hence, for $v \in S(r)$, it follows from the Sobolev embedding and $(f 8)$ that

$$
\begin{aligned}
J_{a}(v) & =\frac{1}{2} \int_{\mathbb{R}^{N}}\left(|\nabla v|^{2}+V(x) f^{2}(v)\right) d x-\int_{\mathbb{R}^{N}} G(f(v)) d x \\
& \geq \frac{C_{1}}{2}\|v\|^{2}-\frac{1}{p+1} \int_{\mathbb{R}^{N}} f^{p+1}(v) d x \\
& \geq \frac{C_{1}}{2}\|v\|^{2}-\frac{2^{\frac{p+1}{4}}}{p+1} \int_{\mathbb{R}^{N}}|v|^{\frac{p+1}{2}} d x \\
& \geq \frac{C_{1}}{2}\|v\|^{2}-C_{2}\|v\|^{\frac{p+1}{2}} .
\end{aligned}
$$

Noting that $p>3$, there exist $r, \beta>0$ such that

$$
J_{a}(v) \geq \beta \quad \text { for }\|v\|=r, v \in E .
$$

Now, set $\varphi \in C_{0}^{\infty}\left(\mathbb{R}^{N}\right)$ with $\varphi>0$ and $K=\sup t \varphi \subset \mathbb{R}^{N}$. Then, for $t>0$,

$$
\begin{aligned}
J_{a}(t \varphi)= & \frac{t^{2}}{2} \int_{\mathbb{R}^{N}}|\nabla \varphi|^{2} d x+\frac{1}{2} \int_{\mathbb{R}^{N}} V(x) f^{2}(t \varphi) d x-\frac{1}{p+1} \int_{K \cap[t \varphi>a]} f^{p+1}(t \varphi) d x \\
& +\frac{1}{p+1} \int_{K \cap[t \varphi>a]} a^{p+1} d x+C \operatorname{med}(K) \\
\leq & \frac{t^{2}}{2}\|\varphi\|^{2}-\frac{1}{p+1} \int_{K \cap[t \varphi>a]} f^{p+1}(t \varphi) d x+\frac{1}{p+1} \int_{K \cap[t \varphi>a]} a^{p+1} d x+C \operatorname{med}(K) \\
\leq & \frac{t^{2}}{2}\|\varphi\|^{2}-\frac{C}{p+1} t^{\frac{p+1}{2}} \int_{K \cap\left[t \varphi>\varphi_{0}\right]} \varphi_{0}^{\frac{p+1}{2}} d x+\frac{1}{p+1} \int_{K \cap\left[\varphi_{0}>t \varphi>a\right]} \varphi_{0}^{p+1} d x+C \operatorname{med}(K) \\
\rightarrow & -\infty \text { as } t \rightarrow+\infty,
\end{aligned}
$$

where $\varphi_{0}=\max \{a, 1\}$. Hence for $t_{0}>0$ sufficiently large, we obtain $e=t_{0} \varphi$ satisfying

$$
J_{a}(e)<0 \quad \text { with } e \in E \backslash S_{r}(0) .
$$

Note that $J_{a}(0)=0$, then $J_{a}$ satisfies the mountain pass geometry. It follows from the above lemma and Lemma 2.1 that there exists a sequence $\left\{v_{n}\right\} \subset E$ satisfying

$$
J_{a}\left(v_{n}\right) \rightarrow c_{a} \quad \text { and } \quad m^{J_{a}}\left(v_{n}\right) \rightarrow 0,
$$

where $c_{a}$ is the mountain pass level of the functional $J_{a}$.

Next, we will prove that $\left\{v_{n}\right\}$ given in (3.7) is bounded in $E$.

Lemma 3.3 The sequence $\left\{v_{n}\right\}$ is bounded in $E$.

Proof By (3.7) we have

$$
J_{a}\left(v_{n}\right) \rightarrow c_{a} \quad \text { and } \quad m^{J_{a}}\left(v_{n}\right) \rightarrow 0 .
$$


Let $\left\{v_{n}^{*}\right\} \subset E^{*}$ satisfying $m^{J a}\left(v_{n}\right)=\left\|v_{n}^{*}\right\|_{E^{*}}$ and

$$
v_{n}^{*}=Q_{1}^{\prime}\left(v_{n}\right)-\gamma_{n}
$$

where $\gamma_{n} \subset \partial Q_{2}\left(v_{n}\right)$. Then

$$
\left\langle v_{n}^{*}+\gamma_{n}, v_{n}\right\rangle=\left\langle Q_{1}^{\prime}\left(v_{n}\right), v_{n}\right\rangle=\int_{\mathbb{R}^{N}}\left|\nabla v_{n}\right|^{2} d x+\int_{\mathbb{R}^{N}} V(x) f\left(v_{n}\right) f^{\prime}\left(v_{n}\right) v_{n} d x
$$

Once we have $0 \leq(p+1) G(f(v)) \leq v g(f(v)) f^{\prime}(v)$, it follows that

$$
Q_{2}\left(v_{n}\right)=\int_{\mathbb{R}^{N}} G\left(f\left(v_{n}\right)\right) d x \leq \frac{1}{p+1} \int_{\mathbb{R}^{N}} v_{n} \underline{g}\left(f\left(v_{n}\right)\right) f^{\prime}\left(v_{n}\right) d x .
$$

By Proposition 2.2, one has

$$
\underline{g}\left(f\left(v_{n}\right)\right) f^{\prime}\left(v_{n}\right) \leq \gamma_{n}(x) \leq \bar{g}\left(f\left(v_{n}\right)\right) f^{\prime}\left(v_{n}\right) \quad \text { a.e. in } \mathbb{R}^{N}
$$

leading to

$$
\underline{g}\left(f\left(v_{n}\right)\right) f^{\prime}\left(v_{n}\right) v_{n} \leq \gamma_{n}(x) v_{n} \quad \text { a.e. in } \mathbb{R}^{N},
$$

which means that

$$
\int_{\mathbb{R}^{N}} \underline{g}\left(f\left(v_{n}\right)\right) f^{\prime}\left(v_{n}\right) v_{n} d x \leq \int_{\mathbb{R}^{N}} \gamma_{n}(x) v_{n} d x=\left\langle\gamma_{n}, v_{n}\right\rangle .
$$

Hence

$$
Q_{2}\left(v_{n}\right) \leq \frac{1}{p+1} \int_{\mathbb{R}^{N}} v_{n} \underline{g}\left(f\left(v_{n}\right)\right) f^{\prime}\left(v_{n}\right) d x \leq \frac{1}{p+1}\left\langle\gamma_{n}, v_{n}\right\rangle .
$$

From (3.8) and (3.9) we have

$$
\begin{aligned}
c_{a}+o_{n}(1)= & J_{a}\left(v_{n}\right)-\frac{1}{p+1}\left\langle v_{n}^{*}, v_{n}\right\rangle \\
= & \frac{1}{2} \int_{\mathbb{R}^{N}}\left|\nabla v_{n}\right|^{2} d x-\frac{1}{p+1} \int_{\mathbb{R}^{N}}\left|\nabla v_{n}\right|^{2} d x \\
& +\frac{1}{2} \int_{\mathbb{R}^{N}} V(x) f^{2}\left(v_{n}\right) d x-\frac{1}{p+1} \int_{\mathbb{R}^{N}} V(x) f\left(v_{n}\right) f^{\prime}\left(v_{n}\right) v_{n} d x \\
& -\int_{\mathbb{R}^{N}} G\left(f\left(v_{n}\right)\right) d x+\frac{1}{p+1}\left\langle\gamma_{n}, v_{n}\right\rangle \\
\geq & \left(\frac{1}{2}-\frac{1}{p+1}\right) \int_{\mathbb{R}^{N}}\left(\left|\nabla v_{n}\right|^{2}+V(x) f^{2}\left(v_{n}\right)\right) d x,
\end{aligned}
$$

which means that $\left\|v_{n}\right\|_{0}$ is bounded. Using the same arguments used in [57, Lemma 2.1] we can obtain that $\left\|v_{n}\right\|$ is bounded in $E$, which completes the proof. 
The following lemma is a key point in our analysis because the functional $Q_{2}$ is not compact. For each $R>0$, let $Q_{2, R}: L^{p+1}\left(B_{R}(0)\right) \rightarrow \mathbb{R}$ be the function

$$
Q_{2, R}(v)=\int_{B_{R}(0)} G(f(v)) d x .
$$

Furthermore, for each $\varphi \in L^{p+1}\left(B_{R}(0)\right)$, define the function $\tilde{\varphi} \in L^{p+1}\left(\mathbb{R}^{N}\right)$ by

$$
\tilde{\varphi}(x)= \begin{cases}\varphi(x), & x \in B_{R}(0), \\ 0, & x \in B_{R}^{c}(0) .\end{cases}
$$

Lemma 3.4 Let $\left\{v_{n}\right\} \subset E$ with $v_{n} \rightarrow v$ in $E$ and $\gamma_{n} \subset \partial Q_{2}\left(v_{n}\right)$ with $\gamma_{n} \rightarrow \gamma_{0}$ in $L^{\frac{p+1}{p}}\left(\mathbb{R}^{N}\right)$.

Then

$$
\gamma_{0}(x) \in\left[\underline{g}(f(v)) f^{\prime}(v), \bar{g}(f(v)) f^{\prime}(v)\right] \text { a.e. in } \mathbb{R}^{N} .
$$

Proof Firstly, we denote by $v_{n, R}, \gamma_{n, R}, v_{R}$ and $\gamma_{0, R}$ the restriction of the functions $v_{n}, \gamma_{n}, v$ and $\gamma_{0}$ to $B_{R}(0)$. For $\forall \varphi \in L^{p+1}\left(B_{R}(0)\right)$, from a simple computation one has

$$
\int_{B_{R}(0)} \gamma_{n, R} \varphi d x=\int_{\mathbb{R}^{N}} \gamma_{n} \tilde{\varphi} d x
$$

and

$$
Q_{2, R}^{0}\left(v_{n, R}, \varphi\right)=Q_{2}^{0}\left(v_{n, R}, \tilde{\varphi}\right)
$$

Noting that

$$
\int_{\mathbb{R}^{N}} \gamma_{n} \tilde{\varphi} d x \leq Q_{2}^{0}\left(v_{n, R}, \tilde{\varphi}\right)
$$

we obtain

$$
\int_{B_{R}(0)} \gamma_{n, R} \varphi d x \leq Q_{2, R}^{0}\left(v_{n, R}, \varphi\right), \quad \forall \varphi \in L^{p+1}\left(B_{R}(0)\right)
$$

which means

$$
\gamma_{n, R} \in \partial Q_{2, R}\left(v_{n, R}\right)
$$

Recalling that $v_{n, R} \rightarrow v_{R}$ in $L^{p+1}\left(B_{R}(0)\right)$ and $\gamma_{n, R} \rightarrow \gamma_{0, R}$ in $L^{\frac{p+1}{p}}\left(B_{R}(0)\right)$, from Proposition 2.2

$$
\gamma_{0, R} \in \partial Q_{2, R}\left(v_{R}\right)
$$

and so, from Proposition 2.3

$$
\gamma_{0, R}(x) \in\left[\underline{g}\left(f\left(v_{R}(x)\right)\right) f^{\prime}\left(v_{R}(x)\right), \bar{g}\left(f\left(v_{R}(x)\right)\right) f^{\prime}\left(v_{R}(x)\right)\right] \text { a.e. in } B_{R}(0),
$$


or equivalently

$$
\gamma_{0}(x) \in\left[\underline{g}(f(v(x))) f^{\prime}(v(x)), \bar{g}(f(v(x))) f^{\prime}(v(x))\right] \text { a.e. in } B_{R}(0) .
$$

Employing the fact that $R>0$ is arbitrary, we have

$$
\gamma_{0}(x) \in\left[\underline{g}(f(v(x))) f^{\prime}(v(x)), \bar{g}(f(v(x))) f^{\prime}(v(x))\right] \text { a.e. in } R^{N} .
$$

Theorem 3.1 Suppose that $c_{a}<c_{\infty}$, where $c_{\infty}$ is the mountain pass level associated with the functional

$$
J_{a}(v)=\frac{1}{2} \int_{\mathbb{R}^{N}}|\nabla v|^{2} d x+\frac{1}{2} \int_{\mathbb{R}^{N}} V_{\infty} f^{2}(v) d x-\frac{1}{p+1} \int_{\mathbb{R}^{N}} f^{p+1}(v) d x, \quad \forall v \in E .
$$

Then, problem (3.2) has at least one nontrivial solution.

Proof From Lemma 3.2 and Lemma 2.1, there exists a sequence $\left\{v_{n}\right\} \subset E$ satisfying

$$
J_{a}\left(v_{n}\right) \rightarrow c_{a} \quad \text { and } \quad m^{J_{a}}\left(v_{n}\right) \rightarrow 0 .
$$

By using standard arguments, we can assume, without loss of generality, that $\left\{v_{n}\right\}$ is bounded in $E$ and $v_{n}(x) \geq 0$ for all $x \in \mathbb{R}^{N}$. Then there exists $v \in E$ such that, passing to a subsequence if necessary,

$$
v_{n} \rightarrow v \quad \text { in } E
$$

and

$$
v_{n} \rightarrow v \quad \text { in } L_{\mathrm{loc}}^{q}\left(\mathbb{R}^{N}\right) \text { for } q \in\left[1,2^{*}\right) .
$$

Claim 1 The weak limit $v$ is nontrivial.

In fact, if $v \equiv 0$, the limit $v_{n} \rightarrow 0$ in $E$ does not hold as $c_{a}>0$. From Lions lemma [58], there exist $\left\{y_{n}\right\} \subset \mathbb{R}^{N}$ and $\alpha, r>0$ satisfying

$$
\liminf _{n \rightarrow \infty} \int_{B_{r}\left(y_{n}\right)}\left|v_{n}\right|^{2} d x \geq \alpha>0 .
$$

Since we are assuming $v=0$, from the Sobolev embedding theorem we obtain that $\left\{y_{n}\right\}$ is unbounded. Now set

$$
w_{n}(x)=v_{n}\left(x+y_{n}\right) .
$$

Employing the boundedness of $\left\{v_{n}\right\}$ in $E$, we infer that $\left\{w_{n}\right\}$ is bounded in $E$. Thus, there exist $w \in E \backslash\{0\}$ and subsequence of $\left\{w_{n}\right\}$, still denote by itself, such that

$$
w_{n} \rightarrow w \text { in } E
$$


and

$$
w_{n} \rightarrow w \text { in } L_{\mathrm{loc}}^{q}\left(\mathbb{R}^{N}\right)
$$

$1 \leq s$ if $N=1,2$ and $1 \leq q<2^{*}$ if $N \geq 3$.

Set $\psi \in C_{0}^{\infty}\left(\mathbb{R}^{N}\right)$ satisfying $\psi(x)=1$ for $x \in B_{1}(0), \psi(x)=0$ for $x \in B_{2}^{c}(0), 0 \leq \psi(x) \leq 1$ and $\psi_{R}(x)=\psi\left(\frac{x}{R}\right)$ for $R>0$. Then, there exists $v_{n}^{*} \in \partial J_{a}\left(v_{n}\right)$ such that

$$
\left\langle v_{n}^{*},\left(\psi_{R} w_{n}\right)\left(\cdot-y_{n}\right)\right\rangle=o_{n}(1)
$$

as the sequence $\left\{\left(\psi_{R} w_{n}\right)\left(\cdot-y_{n}\right)\right\}$ is bounded in $E$. Hence

$$
\begin{gathered}
\int_{\mathbb{R}^{N}}\left(\nabla v_{n} \nabla\left(\psi_{R} w_{n}\right)\left(x-y_{n}\right)+V(x) f\left(v_{n}\right) f^{\prime}\left(v_{n}\right)\left(\psi_{R} w_{n}\right)\left(x-y_{n}\right)\right) d x \\
=\int_{\mathbb{R}^{N}} \gamma_{n}\left(\psi_{R} w_{n}\right)\left(x-y_{n}\right) d x+o_{n}(1),
\end{gathered}
$$

where $\gamma_{n} \in \partial Q_{2}\left(v_{n}\right)$, and so

$$
\begin{aligned}
& \int_{B_{2 R}}\left(\left|\nabla w_{n}\right|^{2} \psi_{R}+V\left(x+y_{n}\right) f\left(w_{n}\right) f^{\prime}\left(w_{n}\right) w_{n} \psi_{R}\right) d x \\
& \quad+\int_{B_{2 R}} w_{n} \nabla w_{n} \nabla \psi_{R} d x \leq \int_{B_{2 R}} f^{p}\left(w_{n}\right) f^{\prime}\left(w_{n}\right) w_{n} \psi_{R} d x .
\end{aligned}
$$

By Fatou's lemma, we have

$$
\begin{aligned}
& \left.\int_{B_{2 R}}\left(|\nabla w|^{2} \psi_{R}\right)+V_{\infty} f(w) f^{\prime}(w) w \psi_{R}\right) d x \\
& +\int_{B_{2 R}} w \nabla w \nabla \psi_{R} d x \leq \int_{B_{2 R}} f^{p}(w) f^{\prime}(w) w \psi_{R} d x .
\end{aligned}
$$

Passing to the limit of $R \rightarrow+\infty$, from the above inequality one deduces that

$$
\int_{\mathbb{R}^{N}}\left(|\nabla w|^{2}+V_{\infty} f(w) f^{\prime}(w) w\right) d x \leq \int_{\mathbb{R}^{N}} f^{p}(w) f^{\prime}(w) w d x
$$

Once we have $w \neq 0$, there exists $t>0$ such that $t w \in \mathscr{N}_{\infty}$, where $\mathscr{N}_{\infty}$ is the Nehari manifold associated with $J_{\infty}$ defined by

$$
\mathscr{N}_{\infty}=\left\{v \in E \backslash\{0\}: J_{\infty}^{\prime}(v) v=0\right\}
$$

Then

$$
\int_{\mathbb{R}^{N}}\left(t^{2}|\nabla v|^{2}+V_{\infty} f(t v) f^{\prime}(t v) t v\right) d x=\int_{\mathbb{R}^{N}} f^{p}(t v) f^{\prime}(t v) t v d x
$$

i.e.,

$$
\int_{\mathbb{R}^{N}}\left(|\nabla v|^{2}+V_{\infty} \frac{f(t v) f^{\prime}(t v)}{t v} v^{2}\right) d x=\int_{\mathbb{R}^{N}} \frac{f^{p}(t v) f^{\prime}(t v)}{t v} v^{2} d x
$$


Note that

$$
\begin{aligned}
{\left[\frac{f^{p}(s) f^{\prime}(s)}{s}\right]^{\prime} } & =\frac{\left[p f^{p-1}(s) f^{\prime 2}(s)+f^{p}(s) f^{\prime \prime}(s)\right] s-f^{p}(s) f^{\prime}(s)}{s^{2}} \\
& =\frac{\left[p f^{p-1}(s) f^{\prime 2}(s)-2 f^{p+1}(s) f^{\prime 4}(s)\right] s-f^{p}(s) f^{\prime}(s)}{s^{2}} \\
& =\frac{f^{p-1}(s) f^{\prime}(s)\left[p f^{\prime}(s) s-2 f^{2}(s) f^{\prime 3}(s) s-f(s)\right]}{s^{2}} \\
& \geq f^{p-1}(s) f^{\prime}(s)\left[(p-1) f^{\prime}(s) s-f(s)\right] \\
& \geq f^{p}(s) f^{\prime}(s)\left(\frac{p-1}{2}-1\right) \\
& >0 \quad \text { for } s>0
\end{aligned}
$$

and

$$
\begin{aligned}
{\left[\frac{f(s) f^{\prime}(s)}{s}\right]^{\prime} } & =\frac{s f^{\prime 2}(s)+f(s) f^{\prime \prime}(s) s-f(s) f^{\prime}(s)}{s^{2}} \\
& =\frac{f^{\prime}(s) \varphi_{1}(s)}{s^{2}}
\end{aligned}
$$

where $\varphi_{1}(s)=f^{\prime}(s) s-2 f^{2}(s) f^{\prime 3}(s) s-f(s)$. Since

$$
\begin{aligned}
\varphi_{1}^{\prime}(s) & =f^{\prime \prime}(s) s+f^{\prime}(s)-4 f(s) f^{\prime}(s) s-6 f^{2}(s) f^{\prime 2}(s) f^{\prime \prime}(s) s-2 f^{2}(s) f^{\prime 3}(s)-f^{\prime}(s) \\
& =f(s) f^{\prime 3}(s)\left[-6 f^{\prime}(s) s+12 f^{2}(s) f^{\prime 3}(s) s-2 f(s)\right] \\
& =f(s) f^{\prime 3}(s)\left[-6 f^{\prime}(s) s+\frac{12 f^{2}(s)}{1+2 f^{2}(s)} f^{\prime}(s) s-2 f(s)\right] \\
& \leq-2 f^{2}(s) f^{\prime 3}(s) \\
& <0
\end{aligned}
$$

for $s>0$, it demonstrates $\left(\frac{f(s) f^{\prime}(s)}{s}\right)^{\prime}<0$ for $s>0$. The above inequalities mean that $\frac{f^{\prime}(t v) f(t v)}{t v}$ is a decreasing function and $\frac{f^{p}(t v) f^{\prime}(t v)}{t v}$ is an increasing function. Then from (3.14) and (3.15) we infer that $t \leq 1$.

By virtue of a result found in Willem [59, Theorem 4.2] we have

$$
c_{\infty} \leq \inf _{v \in \mathscr{N}_{\infty}} J_{\infty}(v)
$$

from which it follows that $c_{\infty} \leq J_{\infty}(t v)$. Consequently

$$
\begin{aligned}
c_{\infty} \leq & J_{\infty}(t v)-\frac{1}{p+1} J_{\infty}^{\prime}(t v) t v \\
= & \frac{t^{2}}{2} \int_{\mathbb{R}^{N}}|\nabla v|^{2} d x+\frac{1}{2} \int_{\mathbb{R}^{N}} V_{\infty} f^{2}(t v) d x-\frac{1}{p+1} \int_{\mathbb{R}^{N}} f^{p+1}(t v) d x \\
& -\frac{t^{2}}{p+1} \int_{\mathbb{R}^{N}}|\nabla v|^{2} d x-\frac{1}{p+1} \int_{\mathbb{R}^{N}} V_{\infty} f(t v) f^{\prime}(t v) t v d x
\end{aligned}
$$




$$
\begin{aligned}
& +\frac{1}{p+1} \int_{\mathbb{R}^{N}} f^{p}(t v) f^{\prime}(t v) t v d x \\
\leq & \left(\frac{1}{2}-\frac{1}{p+1}\right) t^{2} \int_{\mathbb{R}^{N}}|\nabla v|^{2} d x+\frac{1}{2} \int_{\mathbb{R}^{N}} V_{\infty} f^{2}(t v) d x \\
& -\frac{1}{p+1} \int_{\mathbb{R}^{N}} V_{\infty} f(t v) f^{\prime}(t v) t v d x .
\end{aligned}
$$

Set $A(s)=\frac{1}{2} f^{2}(s)-\frac{1}{p+1} f(s) f^{\prime}(s) s$. Then

$$
\begin{aligned}
A^{\prime}(s) & =f(s) f^{\prime}(s)-\frac{1}{p+1}\left[f^{\prime 2}(s) s+f(s) f^{\prime \prime}(s) s+f(s) f^{\prime}(s)\right] \\
& =f(s) f^{\prime}(s)-\frac{1}{p+1}\left[f^{\prime 2}(s) s-2 f^{2}(s) f^{\prime 4}(s) s+f(s) f^{\prime}(s)\right] \\
& =f^{\prime}(s)\left[f(s)-\frac{1}{p+1}\left(f^{\prime}(s) s-2 f^{2}(s) f^{\prime 4}(s) s+f(s) f^{\prime}(s)\right)\right] \\
& \geq f^{\prime}(s)\left[\left(1-\frac{1}{p+1}\right) f(s)-\frac{1}{p+1} f^{\prime}(s) s\right] \\
& \geq f^{\prime}(s) f(s)\left(1-\frac{2}{p+1}\right) \\
& >0 \quad \text { for } p>3, s>0 .
\end{aligned}
$$

Since $t \leq 1$, we have

$$
\begin{aligned}
c_{\infty} \leq & \left(\frac{1}{2}-\frac{1}{p+1}\right) \int_{\mathbb{R}^{N}}|\nabla v|^{2} d x \\
& +\frac{1}{2} \int_{\mathbb{R}^{N}} V_{\infty} f^{2}(v) d x-\frac{1}{p+1} \int_{\mathbb{R}^{N}} V_{\infty} f(v) f^{\prime}(v) v d x .
\end{aligned}
$$

According to Fatou's lemma and the inequality $g(f(s)) f^{\prime}(s) s \geq(p+1) G(f(s))$ for all $s \geq 0$, we derive that

$$
\begin{aligned}
c_{\infty} \leq & \liminf _{n \rightarrow \infty}\left[\left(\frac{1}{2}-\frac{1}{p+1}\right) \int_{\mathbb{R}^{N}}\left|\nabla v_{n}\right|^{2} d x+\frac{1}{2} \int_{\mathbb{R}^{N}} V(x) f^{2}\left(v_{n}\right) d x\right. \\
& \left.-\frac{1}{p+1} \int_{\mathbb{R}^{N}} V(x) f\left(v_{n}\right) f^{\prime}\left(v_{n}\right) v_{n} d x+\int_{\mathbb{R}^{N}}\left(\frac{g\left(f\left(v_{n}\right)\right) f^{\prime}\left(v_{n}\right) v_{n}}{p+1}-G_{H}\left(f\left(v_{n}\right)\right)\right) d x\right] \\
\leq & \liminf _{n \rightarrow \infty}\left[\left(\frac{1}{2}-\frac{1}{p+1}\right) \int_{\mathbb{R}^{N}}\left|\nabla v_{n}\right|^{2} d x+\frac{1}{2} \int_{\mathbb{R}^{N}} V(x) f^{2}\left(v_{n}\right) d x\right. \\
& -\frac{1}{p+1} \int_{\mathbb{R}^{N}} V(x) f\left(v_{n}\right) f^{\prime}\left(v_{n}\right) v_{n} d x+\frac{1}{p+1} \int_{\mathbb{R}^{N}} \gamma_{n} v_{n} d x-\int_{\mathbb{R}^{N}} G\left(f\left(v_{n}\right)\right) d x,
\end{aligned}
$$

that is,

$$
c_{\infty} \leq \liminf _{n \rightarrow \infty}\left[J_{a}\left(v_{n}\right)-\frac{1}{p+1}\left\langle\gamma_{n}, v_{n}\right\rangle\right]=\lim _{n \rightarrow+\infty}\left[J_{a}\left(v_{n}\right)+o_{n}(1)\right]=c_{a}
$$

which is a contraction. Hence $v \geq 0$ and $v \neq 0$. 
In the following, we will prove that $v$ is a solution of problem (3.2). With this aim in mind, we need to show

$$
-\Delta v(x) \in \frac{1}{\sqrt{1+2 f^{2}(v)}}([\underline{g}(f(v)), \bar{g}(f(v))]-V(x) f(v)) \quad \text { a.e. in } \mathbb{R}^{N}
$$

Noting that $\left\{v_{n}\right\} \subset E$ is a (PS) $c_{c_{a}}$ sequence, there exist $v_{n}^{*} \in \partial J_{a}\left(v_{n}\right)$ and $\gamma_{n} \subset \partial Q_{2}\left(v_{n}\right)$ satisfying

$$
\left\|v_{n}^{*}\right\|_{E^{*}} \rightarrow 0
$$

and

$$
\left\langle v_{n}^{*}, y\right\rangle=\int_{\mathbb{R}^{N}}\left(\nabla v_{n} \nabla y+V(x) f\left(v_{n}\right) f^{\prime}\left(v_{n}\right) y\right) d x-\int_{\mathbb{R}^{N}} \gamma_{n} y d x, \quad \forall y \in E,
$$

where $\gamma_{n}(x) \in\left[g\left(f\left(v_{n}\right)\right) f^{\prime}\left(v_{n}\right), \bar{g}\left(f\left(v_{n}\right)\right) f^{\prime}\left(v_{n}\right)\right]$ a.e. in $\mathbb{R}^{N}$. The boundedness of $\left\{v_{n}\right\}$ combined with (3.17) means that $\left\{\gamma_{n}\right\}$ is bounded in $L^{\frac{p+1}{p}}\left(\mathbb{R}^{N}\right)$. Hence, there exist $\gamma_{0} \in L^{\frac{p+1}{p}}\left(\mathbb{R}^{N}\right)$ and a subsequence of $\left\{\gamma_{n}\right\}$, still denoted the same, such that

$$
\gamma_{n} \rightarrow \gamma_{0} \quad \text { in } L^{\frac{p+1}{p}}\left(\mathbb{R}^{N}\right)
$$

It follows from (3.13) and (3.18) that

$$
\int_{\mathbb{R}^{N}}\left(\nabla v \nabla y+V(x) f(v) f^{\prime}(v) y\right) d x=\int_{\mathbb{R}^{N}} \gamma_{0} y d x \quad \forall y \in E .
$$

Furthermore, by Lemma 3.4 we have

$$
\gamma_{0}(x) \in\left[\underline{g}(f(v)) f^{\prime}(v), \bar{g}(f(v)) f^{\prime}(v)\right] \text { a.e. in } \mathbb{R}^{N},
$$

which means that $v$ is a nonnegative weak solution of the following problem:

$$
-\Delta v(x)=\frac{1}{\sqrt{1+2 f^{2}(v)}}\left(\gamma_{0}-V(x) f(v)\right)
$$

Hence (3.19) and (3.20) mean that $v$ is a weak solution of problem (3.2).

Remark 3.1 Due to the fact that $V(x) \geq m$ for all $x \in \mathbb{R}^{N}$, it is easily to verify, by using the Stampacchia theorem, that $\left\{x \in \mathbb{R}^{N}: f(v(x))=a\right\}$ has null measure for $a$ small enough. Thus the weak solution $v$ satisfies

$$
-\Delta v(x)=\frac{1}{\sqrt{1+2 f^{2}(v)}}\left(H(f(v)-a) f^{p}(v)-V(x) f(v)\right) \quad \text { a.e. in } \mathbb{R}^{N}
$$

This is very important in many applications. 


\section{Existence and concentration of solution for (1.4)}

In this part, we define the space

$$
E_{\epsilon}=\left\{u \in H^{1}\left(\mathbb{R}^{N}\right): \int_{\mathbb{R}^{N}} V(\epsilon x)|u|^{2} d x<\infty\right\}
$$

endowed with the norm

$$
\|u\|^{2}=\int_{\mathbb{R}^{N}}\left(|\nabla u|^{2}+V(\epsilon x) u^{2}\right) d x
$$

Similar to (3.2), the dual energy functional associated with (1.4) is defined by

$$
J_{\epsilon, a}(v)=\frac{1}{2} \int_{\mathbb{R}^{N}}|\nabla v|^{2} d x+\frac{1}{2} \int_{\mathbb{R}^{N}} V(\epsilon x) f^{2}(v) d x-\int_{\mathbb{R}^{N}} G(f(v)) d x,
$$

and $c_{\epsilon, a}$ denotes its mountain pass level. Now, we are ready to prove Theorem 1.1.

Proof of Theorem 1.1 Divide the proof into two steps.

Step 1. We firstly show the existence of solutions to problem (1.4). Let $\tilde{v} \in H^{1}\left(\mathbb{R}^{N}\right)$ be a positive ground state solution of the problem

$$
\left\{\begin{array}{l}
-\Delta \tilde{v}=\frac{1}{\sqrt{1+2 f^{2}(\tilde{v})}}\left[f^{p}(\tilde{v})-m f(\tilde{v})\right] \text { in } \mathbb{R}^{N} \\
\tilde{v}>0 .
\end{array}\right.
$$

If $J_{0}: H^{1}\left(\mathbb{R}^{N}\right) \rightarrow \mathbb{R}$ is the energy functional associated with (4.1) given by

$$
J_{0}(v)=\frac{1}{2} \int_{\mathbb{R}^{N}}|\nabla v|^{2} d x+\frac{m}{2} \int_{\mathbb{R}^{N}} f^{2}(v) d x-\frac{1}{p+1} \int_{\mathbb{R}^{N}} f^{p}(v) d x,
$$

we have $J_{0}(\tilde{v})=c_{0}$ and $J_{0}^{\prime}(\tilde{v})=0$, where $c_{0}$ is the mountain pass level of $J_{0}$. Define $\varphi \in$ $C_{0}^{\infty}\left(\mathbb{R}^{N}\right)$ satisfying

$$
0 \leq \varphi(x) \leq 1, \quad \varphi(x)=1 \quad \text { for } \forall x \in B_{1}(0) \quad \text { and } \quad \varphi(x)=0 \quad \text { for } \forall x \in B_{2}^{c}(0)
$$

For each $R>1$, we denote by $\varphi_{R}$ and $\tilde{v}_{R}$ the functions

$$
\varphi_{R}(x)=\varphi\left(\frac{x}{R}\right) \quad \text { and } \quad \tilde{v}_{R}(x)=\varphi_{R}(x) \tilde{v}(x) .
$$

A direct computation shows that

$$
\tilde{v}_{R} \rightarrow \tilde{v} \quad \text { in } H^{1}\left(\mathbb{R}^{N}\right) \text { as } R \rightarrow+\infty .
$$

Thus $\tilde{v}_{R} \neq 0$ for $R$ sufficiently large. By this, there exists $t_{R}>0$ such that

$$
J_{0}\left(t_{R} \tilde{v}_{R}\right)=\max _{t \geq 0} J_{0}\left(t \tilde{v}_{R}\right)
$$


and so

$$
\int_{B_{2 R}}\left[\left|\nabla \tilde{v}_{k}\right|^{2}+\frac{m f\left(t \tilde{v}_{k}\right) f^{\prime}\left(t \tilde{v}_{k}\right) \tilde{v}_{k}^{2}}{t \tilde{v}_{k}}\right] d x=\int_{B_{2 R}} \frac{f^{p}\left(t \tilde{v}_{k}\right) f^{\prime}\left(t \tilde{v}_{k}\right) \tilde{v}_{k}^{2}}{t \tilde{v}_{k}} d x
$$

and

$$
\lim _{R \rightarrow \infty} t_{R}=1
$$

These facts mean that

$$
\hat{v}_{k}=\tilde{v}_{k} t_{k} \rightarrow \tilde{v} \quad \text { in } H^{1}\left(\mathbb{R}^{N}\right) \text { as } R \rightarrow \infty .
$$

Once that $c_{0}<c_{\infty}$ (see [14]), we can choose $\delta, R>0$ such that

$$
c_{0}+\delta<c_{\infty} \text { and } J_{0}\left(\hat{v}_{k}\right)<c_{0}+\frac{\delta}{2},
$$

and $t>0$ satisfying $J_{\epsilon, a}\left(t^{*} \hat{v}_{k}\right)<0$ uniformly for $\epsilon, a>0$ small enough.

Next, we consider $\hat{\gamma}(t)=t\left(t^{*} \hat{v}_{k}\right)$ for $t \in[0,1]$, where $\hat{\gamma} \in \Gamma$. By the definition of $c_{\epsilon, a}$ one has

$$
c_{\epsilon, a} \leq \max _{t \in[0,1]} J_{\epsilon, a}\left(t \hat{v}_{k}\right)=J_{\epsilon, a}\left(\hat{t} \hat{v}_{k}\right)
$$

for some $\hat{t}=\hat{t}(\epsilon, a, R)>0$.

For each given $R>0$, it is obvious that there exist positive constants $A_{1}$ and $A_{2}$ such that $A_{1} \leq \hat{t} \leq A_{2}$ for $\epsilon, a>0$ small enough. Note that $m \leq V(x)$ for all $x \in \mathbb{R}^{N}$. Then

$$
c_{0} \leq c_{\epsilon, a} \leq \max _{t \geq 0} J_{\epsilon, a}\left(t \hat{v}_{k}\right)
$$

Without loss of generality, we suppose that $V(0)=m$. Hence, for each $\zeta>0$, there exists $\epsilon_{0}>0$ such that

$$
0<V(\epsilon x)-m<\zeta \quad \text { for } \epsilon \in\left(0, \epsilon_{0}\right) \text { and } x \in \sup t \hat{v}_{k}=B_{2 R}(0)
$$

from which one deduces that

$$
\int_{\mathbb{R}^{N}} V(\epsilon x) f^{2}\left(\hat{v}_{k}\right) d x<\int_{\mathbb{R}^{N}}(m+\zeta) f^{2}\left(\hat{v}_{k}\right) d x .
$$

By the above inequality we have

$$
\begin{aligned}
c_{\epsilon, a} \leq & J_{0}\left(\hat{t} \hat{v}_{k}\right)+\frac{\zeta}{2} \int_{B_{2 R}} f^{2}\left(\hat{t} \hat{v}_{k}\right) d x+\frac{1}{p+1} \int_{B_{2 k} \cap\left[\hat{t}_{k} \leq a\right]} f^{p+1}\left(\hat{t} \hat{v}_{k}\right) d x \\
& +\frac{1}{p+1} \int_{B_{2 k} \cap\left[\hat{v_{v}}>a\right]} a^{p+1} d x
\end{aligned}
$$

which implies

$$
c_{\epsilon, a} \leq c_{0}+\zeta C_{1}+\frac{\delta}{2}+C_{2} a^{p+1},
$$


where $C_{1}, C_{2}$ do not depend on $\epsilon, a>0$. Hence for $\epsilon, a>0$ small enough we have

$$
c_{\epsilon, a} \leq c_{0}+\frac{\delta}{4}+\frac{\delta}{2}+\frac{\delta}{4} \leq c_{0}+\delta<c_{\infty}
$$

It follows from Theorem 3.1 that problem (1.4) has at least one nontrivial solution for $\epsilon$, $a>0$ sufficiently small.

Step 2. Now, we begin to prove the concentration of the solution. Denote by $v_{\epsilon, a}$ the solution given by step 1 . Thus, there is $\gamma_{\epsilon, a} \in L^{\frac{p+1}{p}}\left(\mathbb{R}^{N}\right)$ such that

$$
-\Delta v_{\epsilon, a}(x)=\frac{1}{\sqrt{1+2 f^{2}\left(v_{\epsilon, a}\right)}}\left(\gamma_{\epsilon, a}-V(x) f\left(v_{\epsilon, a}(x)\right)\right) \text { a.e. in } \mathbb{R}^{N}
$$

with $\gamma_{\epsilon, a}(x) \in\left[g\left(f\left(v_{\epsilon, a}(x)\right)\right) f^{\prime}\left(v_{\epsilon, a}(x)\right), \bar{g}\left(f\left(v_{\epsilon, a}(x)\right)\right) f^{\prime}\left(v_{\epsilon, a}(x)\right)\right]$ a.e. in $\mathbb{R}^{N}$.

Now, fix $\epsilon_{n} \rightarrow 0, a_{n} \rightarrow 0 . v_{n}=v_{\epsilon_{n}, a_{n}}$ and $\gamma_{n}=\gamma_{\epsilon_{n}, a_{n}}$. We are ready to discuss the behavior of the maximum points related to $\left\{v_{n}\right\}$, more precisely, if $y_{n} \in \mathbb{R}^{N}$ denotes a maximum point of $v_{n}$, we will show that

$$
\lim _{n \rightarrow \infty} V\left(\epsilon_{n} y_{n}\right)=m
$$

By just the same method as used in (4.2) and (4.3), we obtain

$$
\lim _{n \rightarrow \infty} c_{\epsilon_{n}, a_{n}}=c_{0}>0 \text {. }
$$

Claim 2 There exist $\left\{z_{n}\right\} \subset \mathbb{R}^{N}$ and $\eta, r>0$ such that

$$
\liminf _{n \rightarrow \infty} \int_{B_{r}\left(z_{n}\right)}\left|v_{n}\right|^{2} d x \geq \eta>0
$$

In fact, if the claim does not hold, from a result due to Lions, one has

$$
\lim _{n \rightarrow \infty} \int_{\mathbb{R}^{N}}\left|v_{n}\right|^{q} d x=0
$$

for $q \in\left(2,2^{*}\right)$. This limit combined with the fact that $v_{n}$ is a solution of (1.4) with $\epsilon=\epsilon_{n}$ and $a=a_{n}$ means that

$$
\lim _{n \rightarrow \infty} c_{\epsilon_{n}, a_{n}}=\lim _{n \rightarrow \infty} J_{\epsilon_{n}, a_{n}}\left(v_{n}\right)=0
$$

which contradicts (4.5).

Claim 3 The sequence $w_{n}=v_{n}\left(\cdot-z_{n}\right)$ is strongly convergent in $H^{1}\left(\mathbb{R}^{N}\right)$. Furthermore,

$$
\lim _{|x| \rightarrow \infty} w_{n}(x)=0
$$

uniformly in $n \in \mathbb{N}$, that is, for $\forall \eta>0$, there exists $R>0$ such that

$$
\left|w_{n}(x)\right|<\eta \quad \forall x \in \mathbb{R}^{N} \backslash B_{R}(0) .
$$


Using the same arguments in Claim 1, we can assume that $\left\{\epsilon_{n} z_{n}\right\}$ is a convergent sequence in $\mathbb{R}^{N}$ with $\epsilon_{n} z_{n} \rightarrow z^{*} \in V^{-1}(m)$. Moreover, we obtain that if $w$ is the weak limit of $\left\{w_{n}\right\}$, then

$$
w_{n} \rightarrow w \quad \text { in } H^{1}\left(\mathbb{R}^{N}\right)
$$

In the following, we prove that

$$
\lim _{|x| \rightarrow \infty} w_{n}(x)=0
$$

The main idea is borrowed from [15]. For $\forall R>0,0<r \leq \frac{R}{2}$. Set $\varphi \in C^{\infty}\left(\mathbb{R}^{N}\right), \varphi \in[0,1]$ with $\varphi(x)=1$ if $|x| \geq R$ and $\varphi=0$ if $|x| \leq R-r$ and $|\nabla \varphi| \leq \frac{2}{r}$. Note that

$$
\left|g(f(v)) f^{\prime}(v)\right| \leq|f(v)|^{p} \leq 2^{\frac{p}{4}}|v|^{\frac{p}{2}} \leq \xi|v|^{2}+C_{\xi}|v|^{2^{*}-1} .
$$

For each $n \in \mathbb{N}$ and $L>0$, set

$$
\begin{aligned}
& v_{L, n}(x)= \begin{cases}v_{n}(x), & v_{n}(x) \leq L, \\
L, & v_{n}(x) \geq L\end{cases} \\
& y_{L, n}=\varphi^{2} v_{L, n}^{2(\beta-1)} v_{n} \quad \text { and } \quad w_{L, n}=\varphi v_{n} v_{L, n}^{\beta-1} \text {, }
\end{aligned}
$$

where $\beta>1$ is to be determined later.

Take $y_{L, n}$ as a test function in (4.4), then

$$
\begin{aligned}
\int_{\mathbb{R}^{N}} \varphi^{2} v_{L, n}^{2(\beta-1)}\left|\nabla v_{n}\right|^{2} d x= & -2(\beta-1) \int_{\mathbb{R}^{N}} \varphi^{2} v_{n} v_{L, n}^{2 \beta-3} \nabla v_{n} \nabla v_{L, n} d x \\
& -2 \int_{\mathbb{R}^{N}} \varphi v_{L, n}^{2(\beta-1)} v_{n} \nabla v_{n} \nabla \varphi d x+\int_{\mathbb{R}^{N}} \gamma_{n} \varphi^{2} v_{L, n}^{2(\beta-1)} v_{n} d x \\
& -\int_{\mathbb{R}^{N}} V(x) f\left(v_{n}\right) f^{\prime}\left(v_{n}\right) \varphi^{2} v_{L, n}^{2(\beta-1)} v_{n} d x
\end{aligned}
$$

For $\xi$ sufficiently small, (4.7) and $\gamma_{n}(x) \leq f^{p}\left(v_{n}\right) f^{\prime}\left(v_{n}\right)$ yield that

$$
\begin{aligned}
\int_{\mathbb{R}^{N}} \varphi v_{L, n}^{2(\beta-1)}\left|\nabla v_{n}\right|^{2} d x \leq & -2 \int_{\mathbb{R}^{N}} \varphi v_{L, n}^{2(\beta-1)} v_{n} \nabla v_{n} \nabla \varphi d x \\
& +\int_{\mathbb{R}^{N}} f^{p}\left(v_{n}\right) f^{\prime}\left(v_{n}\right) \varphi^{2} v_{L, n}^{2(\beta-1)} v_{n} d x \\
\leq & -2 \int_{\mathbb{R}^{N}} \varphi v_{L, n}^{2(\beta-1)} v_{n} \nabla v_{n} \nabla \varphi d x+C_{3} \int_{\mathbb{R}^{N}} \varphi^{2} v_{L, n}^{2(\beta-1)} v_{n}^{2^{*}} d x
\end{aligned}
$$

For each $\epsilon>0$, by Young's inequality we have

$$
\begin{aligned}
\int_{\mathbb{R}^{N}} \varphi^{2} v_{L, n}^{2(\beta-1)}\left|\nabla v_{n}\right|^{2} d x \leq & C_{\xi} \int_{\mathbb{R}^{N}} \varphi^{2} v_{L, n}^{2(\beta-1)} v_{n}^{2^{*}} d x+2 \xi \int_{\mathbb{R}^{N}} \varphi^{2} v_{L, n}^{2(\beta-1)}\left|\nabla v_{n}\right|^{2} d x \\
& +2 C_{\xi} \int_{\mathbb{R}^{N}} v_{n}^{2} v_{L, n}^{2(\beta-1)}|\nabla \varphi|^{2} d x
\end{aligned}
$$


Taking $\xi>0$ sufficiently small, the above inequality becomes

$$
\int_{\mathbb{R}^{N}} \varphi^{2} v_{L, n}^{2(\beta-1)}\left|\nabla v_{n}\right|^{2} d x \leq C \int_{\mathbb{R}^{N}} v_{n}^{2^{*}} \varphi^{2} v_{L, n}^{2(\beta-1)} d x+C \int_{\mathbb{R}^{N}} v_{n}^{2} v_{L, n}^{2(\beta-1)}|\nabla \varphi|^{2} d x
$$

By Hölder's inequality and a Sobolev embedding, we conclude that

$$
\begin{aligned}
\left|w_{L, n}\right|_{2^{*}}^{2} & \leq C\left|\nabla w_{L, n}\right|_{2}^{2} \\
& =C \int_{\mathbb{R}^{N}}\left(\nabla \varphi v_{n} v_{L, n}^{\beta-1}+\varphi \nabla v_{n} v_{L, n}^{\beta-1}+(\beta-1) \varphi v_{n} v_{L, n}^{\beta-2} \nabla v_{L, n}\right)^{2} d x \\
& \leq C \beta^{2}\left[\int_{\mathbb{R}^{N}} v_{n}^{2}|\nabla \varphi|^{2} v_{L, n}^{2(\beta-1)} d x+\int_{\mathbb{R}^{N}} \varphi^{2} v_{L, n}^{2(\beta-1)}\left|\nabla v_{n}\right|^{2} d x\right] .
\end{aligned}
$$

It follows from (4.8) and (4.9) that

$$
\left|w_{L, n}\right|_{2^{*}}^{2} \leq C \beta^{2}\left[\int_{\mathbb{R}^{N}} v_{n}^{2}|\nabla \varphi|^{2} v_{L, n}^{2(\beta-1)} d x+\int_{\mathbb{R}^{N}} \varphi^{2} v_{L, n}^{2(\beta-1)} v_{n}^{2^{*}} d x\right] .
$$

We assert that $v_{n} \in L^{\frac{2^{* 2}}{2}}(|x| \geq R)$ for $R$ large enough and uniformly in $n$. In fact, set $\beta=\frac{2^{*}}{2}$. By virtue of (4.10) one has

$$
\left|w_{L, n}\right|_{2^{*}}^{2} \leq C \beta^{2}\left[\int_{\mathbb{R}^{N}} v_{n}^{2}|\nabla \varphi|^{2} v_{L, n}^{\left(2^{*}-2\right)} d x+\int_{\mathbb{R}^{N}} \varphi^{2} v_{L, n}^{\left(2^{*}-2\right)} v_{n}^{2^{*}} d x\right]
$$

or equivalently

$$
\left|w_{L, n}\right|_{2^{*}}^{2} \leq C \beta^{2}\left[\int_{\mathbb{R}^{N}} v_{n}^{2}|\nabla \varphi|^{2} v_{L, n}^{\left(2^{*}-2\right)} d x+\int_{\mathbb{R}^{N}} v_{n}^{2} \varphi^{2} v_{L, n}^{\left(2^{*}-2\right)} v_{n}^{\left(2^{*}-2\right)} d x\right] .
$$

Using Hölder's inequality with the exponent $\frac{2^{*}}{2}$ and $\frac{2^{*}}{2^{*}-2}$, we see that

$$
\begin{aligned}
\left|w_{L, n}\right|_{2^{*}}^{2} \leq & C \beta^{2}\left[\int_{\mathbb{R}^{N}} v_{n}^{2}|\nabla \varphi|^{2} v_{L, n}^{\left(2^{*}-2\right)} d x\right. \\
& \left.+\left(\int_{\mathbb{R}^{N}}\left(v_{n} \varphi v_{L, n}^{\frac{2^{*}-2}{2}}\right)^{2^{*}} d x\right)^{\frac{2}{2^{*}}}\left(\int_{|x| \geq R / 2} v_{n}^{2^{*}} d x\right)^{\frac{2^{*}-2}{2^{*}}}\right] .
\end{aligned}
$$

From the definition of $w_{L, n}$, we obtain

$$
\begin{aligned}
& \left(\int_{\mathbb{R}^{N}}\left(v_{n} \varphi v_{L, n}^{\frac{2^{*}-2}{2}}\right)^{2^{*}} d x\right)^{\frac{2}{2^{*}}} \\
& \quad \leq C \beta^{2}\left[\int_{\mathbb{R}^{N}} v_{n}^{2}|\nabla \varphi|^{2} v_{L, n}^{\left(2^{*}-2\right)} d x+\left(\int_{\mathbb{R}^{N}}\left(v_{n} \varphi v_{L, n}^{\frac{2^{*}-2}{2}}\right)^{2^{*}} d x\right)^{\frac{2}{2^{*}}}\left(\int_{|x| \geq R / 2} v_{n}^{2^{*}} d x\right)^{\frac{2^{*}-2}{2^{*}}}\right] .
\end{aligned}
$$

Observing that $v_{n} \rightarrow v$ in $H^{1}\left(\mathbb{R}^{N}\right)$, for $R$ sufficiently large, we infer that

$$
\int_{|x| \geq R / 2} v_{n}^{2^{*}} d x \leq \varepsilon \quad \text { uniformly in } n
$$


Hence

$$
\left(\int_{|x| \geq R}\left(v_{n} v_{L, n}^{\frac{2^{*}-2}{2}}\right)^{2^{*}} d x\right)^{\frac{2}{2^{*}}} \leq C \beta^{2} \int_{\mathbb{R}^{N}} v_{n}^{2} v_{L, n}^{\left(2^{*}-2\right)} d x
$$

or equivalently

$$
\left(\int_{|x| \geq R}\left(v_{n} v_{L, n}^{\frac{2^{*}-2}{2}}\right)^{2^{*}} d x\right)^{\frac{2}{2^{*}}} \leq C \beta^{2} \int_{\mathbb{R}^{N}} v_{n}^{2^{*}} d x \leq M<\infty .
$$

By Fatou's lemma in the variable $L$, one derives

$$
\int_{|x| \geq R} v_{n}^{\frac{2^{* 2}}{2}} d x<\infty
$$

which proves the claim.

Notice that if $\beta=\frac{2^{*}(t-1)}{2 t}$ with $t=\frac{2^{* 2}}{2\left(2^{*}-2\right)}$, then $\beta>1, \frac{2 t}{t-1}<2^{*}$ and $v_{n} \in L^{\frac{2 \beta t}{t-1}}(|x| \geq R-r)$. By (4.10) one has

$$
\left|w_{L, n}\right|_{2^{*}}^{2} \leq c \beta^{2}\left(\int_{R-r \leq|x| \leq R} v_{n}^{2} v_{L, n}^{2(\beta-1)} d x+\int_{R-r \leq|x|} v_{n}^{2^{*}} v_{L, n}^{2(\beta-1)} d x\right)
$$

or equivalently

$$
\left|w_{L, n}\right|_{2^{*}}^{2} \leq c \beta^{2}\left(\int_{R-r \leq|x| \leq R} v_{n}^{2 \beta} d x+\int_{R-r \leq|x|} v_{n}^{2^{*}-2} v_{L, n}^{2 \beta} d x\right) .
$$

Hölder's inequality with exponent $\frac{t}{t-1}$ and $t$ shows that

$$
\begin{aligned}
\left|w_{L, n}\right|_{2^{*}}^{2} \leq & c \beta^{2}\left(\int_{R-r \leq|x| \leq R} v_{n}^{\frac{2 \beta t}{t-1}} d x\right)^{\frac{t-1}{t}}\left(\int_{R-r \leq|x| \leq R} 1 d x\right)^{\frac{1}{t}} \\
& +c \beta^{2}\left(\int_{R-r \leq|x|} v_{n}^{\left(2^{*}-2\right) t} d x\right)^{\frac{1}{t}}\left(\int_{R-r \leq|x|} v_{n}^{\frac{2 \beta t}{t-1}} d x\right)^{\frac{t-1}{t}} .
\end{aligned}
$$

Since $\left(2^{*}-2\right) t=2^{* 2}$, we infer that

$$
\left|w_{L, n}\right|_{2^{*}}^{2} \leq C \beta^{2}\left(\int_{R-r \leq|x|} v_{n}^{\frac{2 \beta t}{t-1}} d x\right)^{\frac{t-1}{t}}
$$

Note that

$$
\begin{aligned}
\left|v_{L, n}\right|_{2^{*} \beta(|x| \geq R)}^{2 \beta} & \leq\left(\int_{|x| \geq R-r} v_{L, n}^{2^{*} \beta} d x\right)^{\frac{2}{2^{*}}} \leq\left(\int_{\mathbb{R}^{N}} \varphi^{2} v_{n}^{2^{*}} v_{L, n}^{2^{*}(\beta-1)} d x\right)^{\frac{2}{2^{*}}} \\
& =\left|w_{L, n}\right|_{2^{*}}^{2} \leq c \beta^{2}\left(\int_{|x| \geq R-r} v_{n}^{\frac{2 \beta t}{t-1}}\right)^{\frac{t-1}{t}}=C \beta^{2}\left|v_{n}\right|_{\frac{2 \beta t}{t-1}(|x| \geq R-r)^{2 \beta}},
\end{aligned}
$$

and therefore, from Fatou's lemma, we obtain

$$
\left|v_{n}\right|_{2^{*} \beta(|x| \geq R)}^{2 \beta} \leq C \beta^{2}\left|\nu_{n}\right|_{\frac{2 \beta t}{t-1}(|x| \geq R-r)}^{2 \beta} .
$$


Choosing $\theta=\frac{2^{*}(t-1)}{2 t}, s=\frac{2 t}{t-1}$, we can show that

$$
\left|v_{n}\right|_{\theta^{m+1} s(|x| \geq R)} \leq C^{\sum_{i=1}^{m} \theta^{-i}} \theta^{\sum_{i=1}^{m} i \theta^{-i}}\left|v_{n}\right|_{2^{*}}(|x| \geq R-r),
$$

which means $\left\|v_{n}\right\|_{L^{\infty}}(|x| \geq R) \leq C\left|v_{n}\right|_{2^{*}(|x| \geq R-r)}$. Applying the convergence of $v_{n} \rightarrow v$ in $H$, given $\epsilon>0$, there is $R>0$ such that

$$
\left\|v_{n}\right\|_{L^{\infty}(|x| \geq R)}<\epsilon \quad \forall n \in \mathbb{N} .
$$

Hence

$$
\lim _{|x| \rightarrow \infty} w_{n}(x)=0 \quad \text { uniformly in } n .
$$

Furthermore, from (4.5) we infer that $\lim _{n \rightarrow \infty}\left\|w_{n}\right\|_{\infty, \mathbb{R}^{N}}>0$ and there exist $\delta^{*}>0$ and $n_{0} \in \mathbb{N}$ such that

$$
\left\|w_{n}\right\|_{\infty, \mathbb{R}^{N}} \geq \delta^{*}, \quad \forall n \geq n_{0}
$$

Choose $\eta=\frac{\delta^{*}}{2}$ and $R>0$ such that

$$
w_{n}(x)<\frac{\delta^{*}}{2} \quad \forall x \in \mathbb{R}^{N} \backslash B_{R}(0) \text { and } n \in \mathbb{N},
$$

and so, if $y_{n}$ denotes a maximum point of $w_{n}$, we derive

$$
w_{n}\left(y_{n}\right) \geq \delta^{*} \quad \text { and } \quad y_{n} \in B_{R}(0)
$$

Setting $\hat{y}_{n}$ to be the maximum point of $v_{n}$, we have $\hat{y}_{n}=y_{n}+z_{n}$, which means $\epsilon \hat{y}_{n}=\epsilon_{n} y_{n}+$ $\epsilon_{n} z_{n} \rightarrow z^{*}$. From the continuity of the function $V$ one derives

$$
\lim _{n \rightarrow \infty} V\left(\epsilon_{n} \hat{y}_{n}\right)=V\left(z^{*}\right)=m
$$

Thus the proof is completed.

\section{Acknowledgements}

The author would like to thank professor Jianshe Yu and the referees for their helpful suggestions.

Funding

Research are supported by the National Natural Science Foundation of China (Grant No. 11901126), the Hunan Province Natural Science Foundation of China (Grant No. 2017JJ3222), and the Scientific Research fund of Hunan provincial Education Department (18C0809).

Availability of data and materials

Not applicable.

\section{Competing interests}

The author declares that they have no competing interests. 


\section{Publisher's Note}

Springer Nature remains neutral with regard to jurisdictional claims in published maps and institutional affiliations.

Received: 6 December 2019 Accepted: 16 March 2020 Published online: 26 March 2020

\section{References}

1. Carrião, P., Lehrer, R., Miyagaki, O.: Existence of solutions to a class of asymptotically linear Schrödinger equations in $\mathbb{R}^{N}$ via the Pohozaev manifold. J. Math. Anal. Appl. 428, 165-183 (2015)

2. Do Ó, J., Severo, U.: Quasilinear Schrödinger equations involving concave and convex nonlinearities. Commun. Pure Appl. Math. 8(2), 621-644 (2009)

3. Severo, U., Gloss, E., Silva, E.: On a class of quasilinear Schrödinger equations with superlinear or asymptotically linear terms. J. Differ. Equ. 263, 3550-3580 (2017)

4. Kurihura, S.: Large-amplitude quasi-solitons in superfluids films. J. Phys. Soc. Jpn. 50, 3262-3267 (1981)

5. Borovskii, A., Galkin, A.: Dynamical modulation of an ultrashort high-intensity laser pulse in matter. J. Exp. Theor. Phys. 77, 313-345 (1983)

6. Brandi, H., Manus, C., Mainfray, G., Lehner, T., Bonnaud, G.: Relativistic and ponderomotive self-focusing of a laser beam in a radially inhomogeneous plasma. Phys. Fluids B 5, 3539-3550 (1993)

7. Chen, X., Sudan, R.: Necessary and sufficient conditions for self-focusing of short ultraintense laser pulse in underdense plasma. Phys. Rev. Lett. 70, 2082-2085 (1993)

8. Makhankov, V., Fedyanin, V:: Nonlinear effects in quasi-one-dimensional modes of condensed matter theory. Phys. Rep. 104, 1-86 (1984)

9. Brüll, L., Lange, H.: Solitary waves for quasilinear Schrödinger equations. Expo. Math. 4, 279-288 (1986)

10. Kosevich, A., Ivanov, B., Kovalev, A.: Magnetic solitons. Phys. Rep. 194, 117-238 (1990)

11. Miyagaki, O., Moreira, S.: Nonnegative solution for quasilinear Schrödinger equations that include supercritical exponents with nonlinearities that are indefinite in sign. J. Math. Anal. Appl. 421, 643-655 (2015)

12. Liu, X., Liu, J., Wang, Z.: Quasilinear elliptic equations via perturbation method. Proc. Am. Math. Soc. 141, 253-263 (2013)

13. Liu, X., Liu, J., Wang, Z:: Quasilinear elliptic equations with critical growth via perturbation method. J. Differ. Equ. 254, 102-124 (2013)

14. Rabinowitz, P.: On a class of nonlinear Schrödinger equations. Z. Angew. Math. Phys. 43, 270-291 (1992)

15. Alves, C., Figuiredo, G.: Existence and multiplicity of positive solutions to a $p$-Laplacian equation in $\mathbb{R}^{N}$. Differ. Integral Equ. 19, 143-162 (2006)

16. Goyal, S., Sreenadh, K.: Existence of multiple solutions of $p$-fractional Laplace operator with sign-changing weight function. Adv. Nonlinear Anal. 4(1), 37-58 (2015)

17. Giacomoni, J., Mukherjee, T., Sreenadh, K.: Doubly nonlocal system with Hardy-Littlewood-Sobolev critical nonlinearity. J. Math. Anal. Appl. 467, 638-672 (2018)

18. Jiao, $\mathrm{F}, \mathrm{Yu}, \mathrm{J}$.: On the existence of bubble-type solutions of nonlinear singular problems. J. Appl. Anal. Comput. 1(2), 229-252 (2011)

19. Cerami, G., Solimini, S., Struwe, M.: Some existence results for superlinear elliptic boundary value problems involving critical exponents. J. Funct. Anal. 69, 289-306 (1986)

20. Brown, K., Zhang, Y.: The Nehari manifold for a semilinear elliptic equation with a sign-changing weight function. J. Differ. Equ. 193, 481-499 (2003)

21. Colin, M., Jeanjean, L.: Solutions for a quasilinear Schrödinger equation: a dual approach. Nonlinear Anal. 56, 213-226 (2004)

22. Moameni, A.: Existence of soliton solutions for a quasilinear Schrödinger equations involving supercritical exponent in $\mathbb{R}^{N}$. Commun. Pure Appl. Anal. 7, 89-105 (2008)

23. Wu, X., Wu, K.: Existence of positive solutions, negative solutions and high energy solutions for quasilinear elliptic equations on $\mathbb{R}^{N}$. Nonlinear Anal., Real World Appl. 16, 48-64 (2014)

24. Bezerra do ó, J., Miyagaki, O., Soares, S.: Soliton solutions for quasilinear Schrödinger equations with critical growth. J. Differ. Equ. 248, $722-744$ (2010)

25. He, X., Qian, A., Zou, W.: Existence and concentration of positive solutions for quasilinear Schrödinger equations with critical growth. Nonlinearity 26, 3137-3168 (2013)

26. Silva, E., Vieira, G.: Quasilinear asymptotically periodic Schrödinger equations with subcritical growth. Nonlinear Anal. 72, 2935-2949 (2010)

27. Wang, Y., Zhang, Y., Shen, Y.: Multiple solutions for quasilinear Schrödinger equations involving critical exponent. Appl. Math. Comput. 216, 849-856 (2010)

28. Fang, X., Szulkin, A.: Multiple solutions for a quasilinear Schrödinger. J. Differ. Equ. 254, 2015-2032 (2013)

29. Cassani, D., do O, J., Moameni, A.: Existence and concentration of solitary waves for a class of quasilinear Schrödinger equations. Commun. Pure Appl. Anal. 9, 281-306 (2010)

30. Gloss, E.: Existence and concentration of positive solution for a quasilinear equation in $\mathbb{R}^{N}$. J. Math. Anal. Appl. 371, 465-484 (2010)

31. Wang, W., Yang, X., Zhao, F.: Existence and concentration of ground states to a quasilinear problem with competing potentials. Nonlinear Anal. 102, 120-132 (2014)

32. Gasinski, L., Papageorgiou, N.S., Petiurenko, A.: Schrödinger Robin problems with indefinite potential and logistic reaction. Complex Var. Elliptic Equ. (2019). https://doi.org/10.1080/17476933.2019.1709968

33. Dhanya, R., Prashanth, S., Tiwari, S., Sreenadh, K.: Elliptic problems in with critical and singular discontinuous nonlinearities. Complex Var. Elliptic Equ. 61, 1668-1688 (2016)

34. Sreenadh, K., Tiwari, S.: Multiple positive solutions of singular and critical elliptic problem in $\mathbb{R}^{2}$ with discontinuous nonlinearities. NoDEA Nonlinear Differ. Equ. Appl. 20, 1831-1850 (2013)

35. Dhanya, R., Prashanth, S., Sreenadh, K., Tiwari, S.: Critical growth elliptic problem in $\mathbb{R}^{2}$ with singular discontinuous nonlinearities. Adv. Differ. Equ. 19, 409-440 (2014)

36. Bahrouni, A., Ounaies, H., Radulescu, V.D.: Bound state solutions of sublinear Schrödinger equations with lack of compactness. Rev. R. Acad. Cienc. Exactas Fís. Nat., Ser. A Mat. 113, 1191-1210 (2019) 
37. Bahrouni, A., Ounaies, H., Radulescu, V.D.: Compactly supported solutions of Schrödinger equations with small perturbation. Appl. Math. Lett. 84, 148-154 (2018)

38. Xue, Y.F., Tang, C.L.: Existence of a bound state solution for quasilinear Schrödinger equations. Adv. Nonlinear Anal. 8, 323-338 (2019)

39. Vétois, J., Wang, S.: Infinitely many solutions for cubic nonlinear Schrödinger equations in dimension four. Adv. Nonlinear Anal. 8, 715-724 (2019)

40. Chang, K.: Variational methods for nondifferentiabe functionals and their applications to partial differential inequalities. J. Math. Anal. Appl. 80, 102-129 (1981)

41. Chang, K.: On the multiple solutions of the elliptic differential equations with discontinuous nonlinear terms. Sci. Sin. 21, 139-158 (1978)

42. Chang, K.: The obstacle problem and partial differential equations with discontinuous nonlinearities. Commun. Pure Appl. Math. 33, 139-158 (1978)

43. Clarke, F.: Optimization and Nonsmooth Analysis. Wiley, New York (1983)

44. Motreanu, D., Rădulescu, V.: Variational and Non-Variational Methods in Nonlinear Analysis and Boundary Value Problems. Kluwer Academic, Boston (2003)

45. Motreanu, D., Pangitoplous, P.: Minimax Theorems and Qualitative Properties of the Solutions of Hemivariational Inequalities. Kluwer Academic, Dordrecht (1998)

46. Gasiński, L., Papageorgiou, N.: Nonsmooth Critical Point Theory and Nonlinear Boundary Value Problems. Chapman \& Hall/CRC Press, Boca Raton (2005)

47. Filippaks, M., Gasiński, L., Papageorgiou, N.: On the existence of positive solutions for hemivariational inequalities driven by the $p$-Laplacian. J. Glob. Optim. 31, 173-189 (2005)

48. Denkowski, Z., Gasiński, L., Papageorgiou, N.: Existence and multiplicity of solutions for semilinear hemivariational inequalities at resonance. Nonlinear Anal. 66, 1329-1340 (2007)

49. Iannizzotto, A., Papageorgiou, N.: Existence of three nontrivial solutions for nonlinear Neumann hemivariational inequalities. Nonlinear Anal. 70, 3285-3297 (2009)

50. Alves, C., Figuiredo, G., Nascimento, R.: On existence and concentration of solutions for an elliptic problem with discontinuous nonlinearity via penalization method. Z. Angew. Math. Phys. 65, 19-40 (2014)

51. Kyritsi, S., Papageorgiou, N.: Multiple solutions of constant sign for nonlinear nonsmooth eigenvalue problems near resonance. Calc. Var. Partial Differ. Equ. 20, 1-24 (2004)

52. Zhang, J., Zhou, Y.: Existence of a nontrivial solutions for a class of hemivariational inequality problems at double resonance. Nonlinear Anal. 74, 4319-4329 (2011)

53. Badiale, M., Tarantello, G.: Existence and multiplicity results for elliptic problems with critical growth and discontinuous nonlinearities. Nonlinear Anal. 29, 639-677 (1997)

54. Chang, K.: Variational methods for nondifferentiabe functionals and their applications to partial differential inequalities. J. Math. Anal. Appl. 80, 102-129 (1981)

55. Alves, C.O., Berone, A.M., Goncalves, J.V.: A variational approach to discontinuous problems with critical Sobolev exponents. J. Math. Anal. Appl. 265, 103-127 (2002)

56. Liu, J., Wang, Y., Wang, Z.: Solutions for quasilinear Schrödinger equation, II. J. Differ. Equ. 187, 473-493 (2003)

57. Zhang, J., Tang, X., Zhang, W.: Infinitely many solutions of quasilinear Schrödinger equation with sign-changing potential. J. Math. Anal. Appl. 420, 1762-1775 (2014)

58. Lions, P:: The concentration-compactness principle in the calculus of variations. The locally compact case II. Ann. Inst. Henri Poincaré, Anal. Non Linéaire 1, 223-283 (1984)

59. Willem, M.: Minimax Theorems. Birkhäuser, Boston (1996)

\section{Submit your manuscript to a SpringerOpen ${ }^{\circ}$ journal and benefit from:}

- Convenient online submission

- Rigorous peer review

- Open access: articles freely available online

- High visibility within the field

Retaining the copyright to your article

Submit your next manuscript at $\gg$ springeropen.com 